5 Gabutti V, Miniero R, Piga A, Incarbone E, Sacchetti L, Balegno G. Behaviour of myeloid precursors in homozygous $\beta$-thalassaemia. Br J Haematol 1980; 45: 599-605.

6 Gabutti V, Piga A, Fortina P, Miniero R, Nicola P. Correlation between transfusion requirement, blood volume, and haemoglobin level in homozygous betathalassaemia. Acta Haematol (Basel) 1980; 64: 103-8.

7 Cavill I, Worwood M, Jacobs A. Internal regulation of iron absorption. Nature 1975; 256 : 328-9.
8 De Alarcon P A, Donovan M E, Forbes G B, Landaw S A, Stockman J A, III. Iron absorption in the thalassemia syndromes and its inhibition by tea. $N$ Engl J Med 1979; $300: 5-8$.

Correspondence to Dr Vilma Gabutti, Clinica Pediatrica, Università di Torino, Piazzale Polonia 94, 10126 Turin, Italy.

Received 5 October 1981

\title{
Elusive blood clots and fluctuating ventricular dilatation after neonatal intraventricular haemorrhage
}

\author{
C-L FAWER AND MALCOLM I LEVENE
}

Department of Paediatrics and Neonatal Medicine, Institute of Child Health, Hammersmith Hospital, London

SUMMARY Two cases are described of a previously unrecognised sequel of posthaemorrhagic ventricular dilatation. The first case documents freely mobile blood clots within the lateral ventricular system, the second variable asymmetry in the size of the dilated lateral ventricle. The unilateral ventricular dilatation depended on which side the infant was lying, the dependent ventricle being considerably larger than the upper one within 4 hours of head turning. Each of these conditions spontaneously resolved with no specific treatment.

Intracranial haemorrhage and consequent posthaemorrhagic dilatation are well-known entities in preterm babies ${ }^{12}$ and real-time ultrasonography has proved to be a safe and accurate method for diagnosing intracerebral haemorrhage and measuring ventricular size. ${ }^{3-5}$

By using real-time ultrasonography routinely we have been able to follow the development of this condition and to note two unusual consequences of posthaemorrhagic dilatation.

\section{Patients}

Case 1. A boy was born at 30 weeks of gestation by spontaneous vaginal vertex delivery. Apgar score was 4 at one minute and 8 at ten minutes. Soon after birth he developed signs of respiratory distress and a chest $x$-ray film showed severe hyaline membrane disease. The baby was electively intubated at 4 hours of life for transporting to Hammersmith Hospital and then mechanically ventilated for 9 days. On arrival he was hypothermic $\left(33^{\circ} \mathrm{C}\right)$ and acidotic (pH 6.89). Two hours later $\mathrm{pH}$ was 7.27 and his condition appeared clinically to be improved. At age 48 hours his general condition deteriorated and he was noted to have grossly abnormal movements and thought clinically to have sustained intraventricular haemorrhage.

The occipitofrontal head circumference grew rapidly from the 10th day of life despite lumbar punctures and a ventriculoatrial shunt was inserted on the 40th day of life. When discharged at age 9 weeks, the infant had a pronounced head lag, abnormal eye movements, and a paralytic squint of the right eye. He was however responsive and both visually and auditorily quite alert.

Ultrasound scans showed bilateral intraventricular haemorrhage to have occurred at about age 72 hours with considerable intraparenchymal extension. Distended ventricles were first noted at 5 days of life and echogenic clots within the ventricles were clearly defined. By the 10th day of life, the posthaemorrhagic dilatation became very pronounced with considerable cerebral compression. Subsequent ultrasound examinations showed that when the infant was 30 days of age, the clots progressively became free within the ventricular system, and were from time to time either clearly visible or totally absent.

In order to determine the movements and the position of the clots in relation to the baby's posture, we performed repeated scans, each time with the infant in a different position (Fig. 1).

When the infant was in the left lateral position, a clot was noted to be lying against the most lateral margin of the left lateral ventricle. The baby was then moved to lie supine, and the clots disappeared, although the most posterior part of the occipital poles could not be adequately visualised. When the 
infant was placed in the prone position, the clot reappeared to lie on the floor of the body of the lateral ventricle. By gently shaking the baby's head, it was possible to displace the clot which drifted slowly away. The shape of the clot seemed on parasagittal view to conform to the cast of the lateral ventricles at the time of the original bleed before dilatation occurred. During a period of 2 to 3

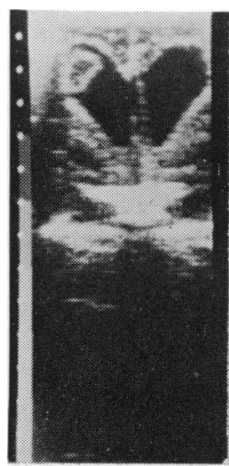

Lateral

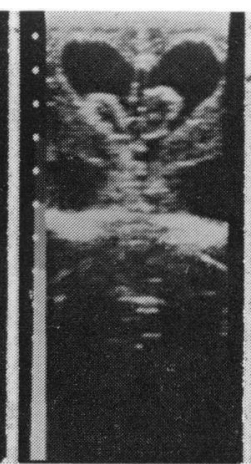

Prone

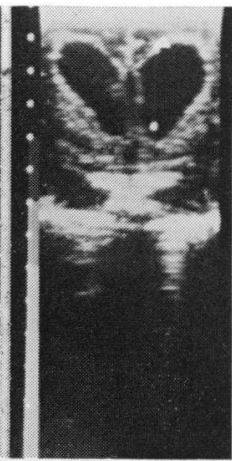

Supine
Fig. 1 Coronal transfontanelle scans showing the position of the mobile blood clots with different positions of the infant's head. (Kranzbühler ADR with 7 MHz transducer.) weeks the clots spontaneously disappeared with no treatment.

Case 2. A boy was born by breech with assisted forceps at 30 weeks' gestation. Apgar score was 4 at one minute, 3 at six minutes, and 9 at nine minutes. He required intubation at delivery and was then mechanically ventilated for 4 days. He was transferred to this hospital at age 12 hours. On the first day of life he was noted to have a distended abdomen and an $x$-ray film showed massive pneumoperitoneum. Peritoneal fluid and blood cultures grew Staphylococcus aureus and Staphylococcus epidermidis. The origin of the peritoneal gas was thought to be from a rectal perforation. Penicillin, gentamicin, and metronidazole were given intravenously and prolonged intravenous parenteral alimentation was undertaken for 3 weeks. The baby was discharged at 39 weeks' gestation and at that time the neurological examination was normal except for a poor auditory response.

Real-time ultrasound on the first day of life showed a large bilateral germinal layer haemorrhage with some downwards extension but no intraparenchymal involvement. From the 6 th to the 19th days of life the ventricles enlarged, and subsequently the dilatation arrested and spontaneously resolved with no specific treatment. On the 16th day of life we
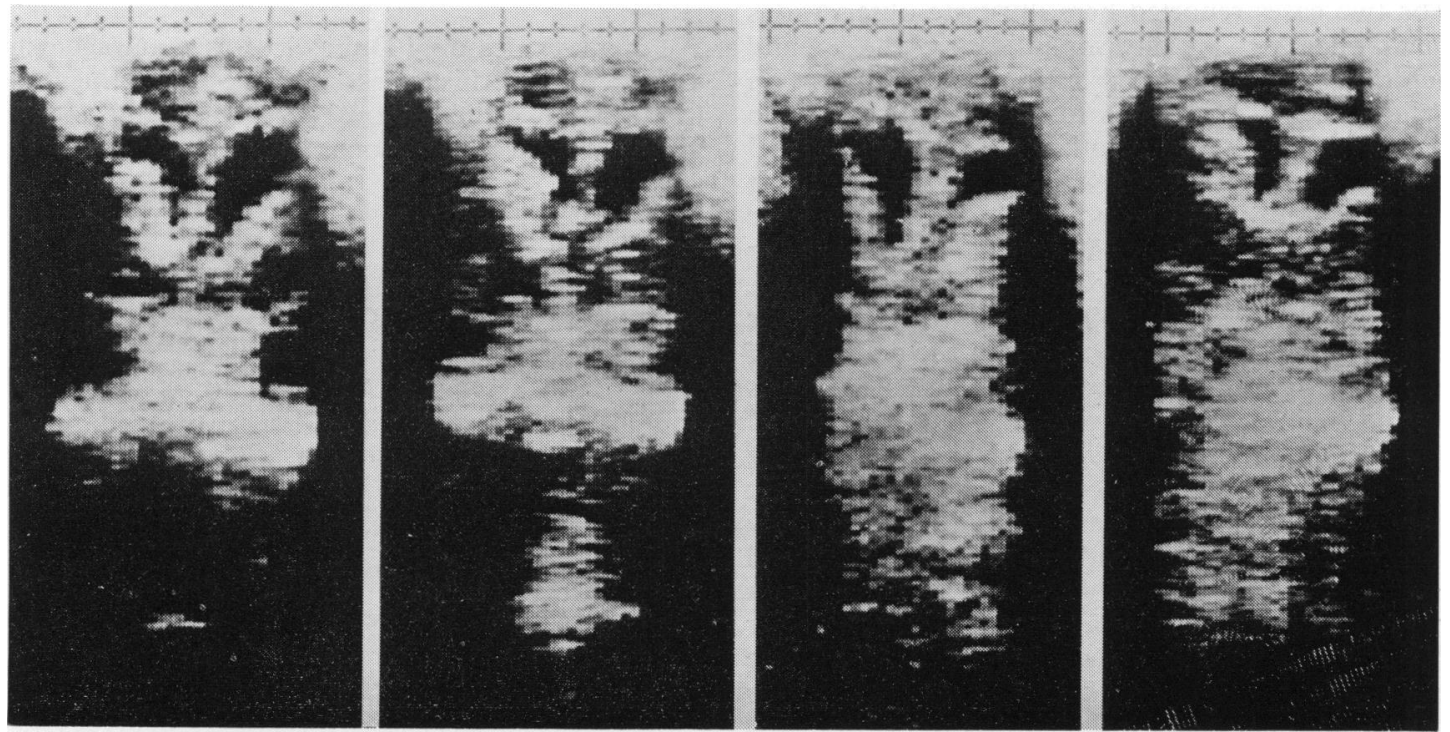

Fig. 2 Coronal transfontanelle scans showing changes in the size of the lateral ventricles with position of the head.The scan on the extreme left is with the infant lying on his right side and the right ventricle is dilated. The progression of scans towards the right shows the change in size of the left lateral ventricle after 2 hours of the infant lying on its left side. The scan on the extreme right demonstrates this reversal back again 4 hours after the infant's head has again been turned to the right. (Kranzbühler ADR with 5 MHz transducer.) 
noticed a striking disparity between the size and shape of the bodies of the two lateral ventricles (Fig. 2). We were able to demonstrate by sequential scans that the variations were dependent on the baby's posture.

When the infant was lying on his left side, the left ventricle was considerably larger than the right. The infant was then turned to lie on his right side for the next 2 hours and the right lateral ventricle enlarged, so that they were roughly equal in size. After a further 2 hours of lying on his right side, the body of the right lateral ventricle became enlarged and the left side became much smaller. This pattern of change in ventricular size could then be repeated by turning the baby back through these positions. This sequence was only noted during one week when the ventricles were particularly dilated, and disappeared as the ventricles reduced in size.

\section{Discussion}

The introduction of computerised $x$-ray tomography and, more recently, of real-time cerebral ultrasonography has made possible the study of the natural history of intraventricular haemorrhage and consequent changes in ventricular size. Ventricular dilatation occurred in nearly $40 \%$ of infants followed sequentially after intracranial haemorrhage but frank clinical hydrocephalus was much less common. ${ }^{2}$ The two cases reported here illustrate observations made when frequent ultrasound scans of the cerebral ventricles are performed after haemorrhage. We believe such observations to be important for two reasons. The first is that both are as yet unrecognised and appear to be unique. The second reason is that such conditions may be thought to have therapeutic implications. Freely mobile clot in the ventricular system might conceivably cause sudden and complete obstruction to cerebrospinal fluid (CSF) flow at either the foramen of Monro, or at the aquedect of Sylvius with severe neurological sequelae. Case 1 was nursed mainly supine or with his head in a lateral position in order to prevent this possibility. The initial germinal layer haemorrhage in this infant burst into the ventricles and thrombus presumably developed through the entire extent of both lateral ventricles. This caused obstruction to CSF flow as a result of the haemorrhage, and progressive ventricular dilatation occurred with no further bleeding. The clots, originally the shape of the ventricles, then became detached and floated freely within the considerably dilated lateral ventricles. The fact that the clots spontaneously disappeared with time is important in case surgical removal is seriously considered.

In Case 2, pronounced irregularity in the size of the lateral ventricles may be due to partial obstruction at the foramen of Monro. This was clearly not the case; in fact, free communication of CSF through this structure must clearly have been possible. Lack of therapeutic intervention again appeared to be the appropriate management as eventually there was complete resolution.

An explanation for this phenomenon is provided by consideration of the relative changes in intracranial pressure. The normal pressure within the ventricular system in an infant of this gestation is probably 7 to $10 \mathrm{~cm}$ of water ${ }^{6}$ and the distance between the midpoints of the bodies of the lateral ventricles was measured at $1.5 \mathrm{~cm}$. If the system were freely communicating and the brain of low density, this represents a pressure difference of 15 to $20 \%$ between the midpoints of the two ventricles when the infant's head was on the side. This increased hydrostatic pressure in the dependent ventricle might be enough to cause distension and distortion of it with contraction of the upper one.

The point in reporting these cases is to stress that methods-such as ultrasound which can be used repeatedly and without risk to visualise previously unstudied structures-often raise more questions than they provide answers. It may be tempting to perform therapeutic manoeuvres to restore the status quo but we believe that a more restrained 'wa通 and see' attitude is often a wiser course of action.

We thank Professor V Dubowitz for encouragement and help with this work, and the Department of Medical Illustration, Royal Postgraduate Medical School, for the illustrations.

\section{References}

1 Burstein J, Papile L, Burstein R. Intraventricular hemorrhage and hydrocephalus in premature newborns: a prospective study with CT. $A J R$ 1979; 132: $631-5$.

2 Levene M I, Starte D. A longitudinal study of posthaemorrhagic ventricular dilatation in the newborn. Arch Dis Child 1981; 56: 905-10.

3 Pape K E, Blackwell R, Cusick G, et al. Ultrasound detection of brain damage in preterm infants. Lancet 1979 ; i: $1261-4$.

4 Levene M I, Wigglesworth J S, Dubowitz V. Cerebral 윽 structure and intraventricular haemorrhage in the $D$ neonate: a real-time ultrasound study. Arch Dis Child 1981 ; 56: 416-24.

5 Levene M I. Measurement of the growth of the lateral $N$ ventricles in preterm infants with real-time ultrasound. Arch Dis Child 1981; 56: 900-4.

6 Donn S M, Philip A G S. Early increase in intracranial N pressure in preterm infants. Pediatrics $1978 ; 61$ : 904-7.

Correspondence to Dr M Levene, Institute of Child Health, 6 Department of Paediatrics and Neonatal Medicine, $\frac{\tau}{\mathbb{D}}$ Hammersmith Hospital, Du Cane Road, London W12 0HS. \&

Received 1 September 1981 